\title{
Comparative Study on Methods for Preparation of Gold Nanoparticles
}

\author{
Khalida S. Merza ${ }^{1}$, Hadi D. Al-Attabi ${ }^{2}$, Zaid M. Abbas ${ }^{1}$, Hashim A. Yusr ${ }^{2}$ \\ ${ }^{1}$ Chemistry Department, College of Science, Wassit University, Al Kut, Iraq \\ ${ }^{2}$ Physical Department, College of Science, Wassit University, Al Kut, Iraq \\ Email: \{dr_ksm, hadidawyich, zaid_almalki,hsmphys\}@yahoo.com
}

Received November 3, 2011; revised December 15, 2011; accepted December 25, 2011

\begin{abstract}
The gold nanoparticles were prepared in many methods, differed in reducing and dispersion agents and stability. The first solution was prepared by using potassium bitartrate as reducing agent and Polyethylene glycol as a dispersion factor. We got a solution of colloid gold with wine-red color that has been measured the particle size; it was between 50 200 nanometers. The second gold colloidal solution was prepared by using sodium citrate as a reducing agent and without adding a dispersion agent, the color of the final solution is dark grey, but this solution did not remain stable, deposition happened quickly and crystals separated from the solution. The third one was prepared by using sodium citrate as a reducing agent and the solution was heated to a certain temperature, and the color of the solution became dark red after several minutes. Either the fourth method has been prepared gold colloid solution by using potassium bitartrate as a reductant for the gold ion. We have noted that no reaction under normal conditions, while the reaction by heating the solution to high temperature, and when added poly vinyl pyrrolidone will lead to a solution of colloild gold with dark purple-red color and stable under normal conditions for a long time. We measured the particles size in this experiment; it was less than 100 nanometers. We conclude from the above that all the reducing agent needs to appropriate conditions to complete the process of reduction of gold ion, and to get the nano gold particles, therefore; these particles in the colloid solution need to dispersion factor to the survival of the particles widespread so that the solutions with certain concentrations of poly vinyl pyrrolidone can be proven in the process of stability of the gold colloid solution.
\end{abstract}

Keywords: Nanotechnology; Gold Colloidal Solution; Nanoparticles

\section{Introduction}

Nanotechnology (NT) is the production and use of materials with purposely engineered features close to the atomic or molecular scale and involves the investigation and design of materials or devices at the atomic and molecular levels.

Two main approaches are used in nanotechnology. In the "bottom-up" approach, materials and devices are built from molecular components which assemble themselves chemically by principles of molecular recognition. In the "top-down" approach, nano-objects are constructed from larger entities without atomic-level control. The original meaning of "nanotechnology" refers to the ability to build components from the bottom up, using techniques and tools that are constantly being developed to become integrated, and whose performance is high. This technique leads to provide the ability to create materials, devices and systems with new functions and characteristics of substance. As a result to that it has large applications in the areas of manufacturing and production [1,2]. Nanoparticles are much smaller than those used in chemical and industrial applications, where can be small enough to enable them to enter into a living cell easily. In studying of the elements and compounds, it is found that is different when it is on the nano scale. Nanotechnology is a wide range of flourishing scientific research. Manufacturing of most nanoparticles during industrial processes as by products. Researchers at the University of MissouriColumbia discovered a method to prepare nanoparticles by the addition of gold salts to a soybean-water mixture. Through the interaction of chemicals in soybean with gold salts has been producing stable gold nano particles with no toxic by-products.

The researchers found a practical process to connect the phytochemicals to cancer cells directly through the gold particles and thus kill the cancer cells.

The researchers selected gold for their research because it is very stable, chemically, therefore, it is less likely to lead to side effects in the body. They said that the production process, they have pioneered, could easily be replicated on a larger scale.The gold in $\mathrm{HAuCl}_{4.3} \mathrm{H}_{2} \mathrm{O}$ complex as $\mathrm{Au}^{3+}$ ions are reduced to neutral gold atoms, 
where citrate ions act as both a reducing agent and a capping agent. The formation of gold nanoparticles can be detected by observing the change in the solution to the red color.The presence of the colloidal suspension can be detected by the reflection of a laser beam from the particles. The layer of absorbed citrate anions on the surface of the nanoparticles keep them separated. Switching to a smaller anion allows the particles to approach more closely and another color change is observed [3].

Colloidal gold is a suspension of sub-micrometer-sized particles of goldin an aqueous medium. The color of the solution is either red when the particles size less than 100 $\mathrm{nm}$ or a dirty yellowish color for larger particles. Due to the optical and electronic properties of gold nanoparticles is the area of research which have a very broad [4].

Properties and applications of colloidal gold nanoparticles depend upon shape. For example, rod like particles have both transverse and longitudinal absorption peak, and anisotropy of the shape affects their self-assembly.

Gold nanoparticles are prepared by the reduction of chloroauric acid $\left(\mathrm{H}\left[\mathrm{AuCl}_{4}\right]\right)$, after addition of reducing agent the solution is rapidly stirred leads to the reduction of gold ion $\mathrm{Ag}^{3+}$ to neutral gold atom and the continuation of the operation will turn out all the gold ions to neutral atoms and the solution becomes supersaturated. If the solution is stirred vigorously enough that will lead to the formation of nanoparticles uniform in size. To avoid the particles from aggregating must use stabilizing agent or to spread particles of each other. This agent can be functionalized with various organic ligands to create organicinorganic hybrids with advanced functionality. It can also be synthesised by laser ablation [5].

Chemical reduction methods have been extensively used in the preparation of nanoparticles because these methods are simple and cheap and can be used to prepare large quantities of nanoparticles. Series of chemical reductions have been utilized for the preparation of noble metal Nanoparticles, which include $\mathrm{H}_{2}, \mathrm{NaBH}_{4}, \mathrm{~N}_{2} \mathrm{H}_{2}$, $\mathrm{NH}_{2} \mathrm{OH},\left(\mathrm{CH}_{3}\right) 2 \mathrm{NH} \cdot \mathrm{BH}_{3}$, ethanol, ethylene glycol, citrate, formamide, ormaldehyde or Tollens reagents, ascorbic acid, and polyaniline or o-anisidine.

The aim of this research is to know the best, simple and efficient method to prepare colloidal gold and itsparticle size located within the nanoscale, for using it in other applications.

\section{Experimental}

\subsection{Materials}

Hydrogen tetrachlorourate (III) trihydrate $\left(\mathrm{HAuCl}_{4} \cdot 3 \mathrm{H}_{2} \mathrm{O}\right)$ and poly vinyl pyrrolidone (PVP) were purchased from Aldrich. Potassium bitartrate, polyethylene glycol (PEG) and sodium citrate were purchased from Merck. For the preparation of mixture solution, deionized water was used.

\subsection{The Methods}

The colloidal forms of gold nanoparticles were prepared by four methods:

First method: nanoparticles were obtained by heating $20 \mathrm{ml}$ potassium bitartrate solution $(0.5 \mathrm{wt} \%)$ with stirring (in water bath) at $60^{\circ} \mathrm{C}$, and $20 \mathrm{ml}$ of an aqueous solution of $\mathrm{HAuCl}_{4}(1.0 \mathrm{Mm})$ containing polyethylene glycol (1.0 wt\%). After 5 min the color of the solution changed from light yellow to wine-red, indicating the formation of Au colloidal nanoparticles.

Second method: the gold nanoparticles were obtained by adding $20 \mathrm{ml}$ of trisodium citrate $(0.5 \mathrm{wt} \%)$ to $20 \mathrm{ml}$ of an aqueous solution of $\mathrm{HAuCl}_{4}(0.1 \mathrm{Mm})$ the solution became a dark-grey after $20 \mathrm{~min}$.

Third method: included preparation of gold nanoparticles by heating $20 \mathrm{ml}$ of $\mathrm{HAuCl} 4(1.0 \mathrm{Mm})$ on a stirring hot plate and bring the solution just to boil with stirring. To the boiling solution, add $2 \mathrm{ml}$ of $1 \%$ sodium citrate solution. When the solution became dark red it removed from heat.

Fourth method: The Au colloidal solution was prepared by heating $25 \mathrm{ml}$ of potassium bitartrate solution $(0.5 \%$ and $1 \%)$ with stirring and $25 \mathrm{ml}$ of $\mathrm{HAuCl}_{4}$ solution (1.0 $\mathrm{Mm})$ containing PVP solution (1.5 wt\%) was added. The color of the solution changed to dark purple red after 5 $\min$.

\section{Results and Discussion}

The formation of gold colloidal nanoparticles can be observed by change in color of the solutions. Potassium bitartrate is a potassium acid salt of tartaric acid, a carboxylic acid, it is used for preventing sugar syrups from crystallizing and for reducing discoloration of boiled vegetables [6]. Potassium bitartrate is a weak reductant, relative to sodium citrate. Sodium citrate can be used as a buffering agent or acidity regulator, because it resists the change in $\mathrm{pH}$. When mixing a solution of sodium citrate with aqueous solution of $\mathrm{HAuCl}_{4}$ the resulting is dark grey solution within minutes and this indicates a reduction reaction happened. In case of addition of potassium bitartrate to the solution of $\mathrm{HAuCl}_{4}$, no change in the color of the resulting solution after an hour of addition and under the same conditions [7].

In the first experiment when using Potassium bitartrateas a reductant and polyethylene glycol as a dispersion, red wine solution was obtained and remained stable in normal conditions the particle size was measured it was between 50 - 200 nanometers as shown in the (Figure 1). In the second and third methods when using sodium citrate as a reductant and without dispersion factor, the color of the resulting solution does not clear that the gold particles is located within the nanoscale. So we did not measure the particle size, because we know that the color 




Figure 1. The range of gold particles size by using atomic force. (PEG as a protective agent).

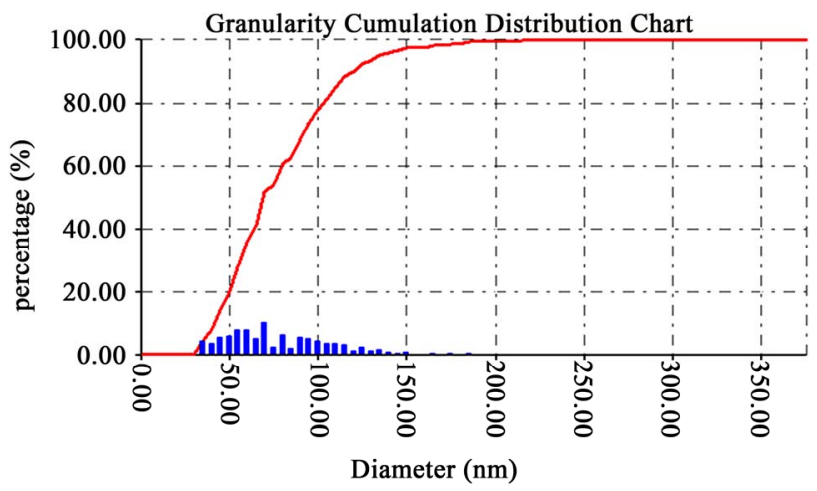

Figure 2. The range of gold particles size by using atomic force. (PVP as a protective agent).

of gold nano particles is red wine or dark purple red. As a result of this experiment shows how important the use of an appropriate reducing agent and the dispersion factor for the survival of a stable solution without clustered particles.

$\mathrm{Au}$ colloidal solution obtained by the reduction of $\mathrm{HAuCl}_{4}$ with potassium bitartrate is unstable in the absence of the stabilizer but when the Au nanoparticles prepared by the reduction of $\mathrm{HAuCl}_{4}$ with potassium bitartrate in the presence of PVP the solution are stable for a long period.

As for the fourth experiment, we used PVP as a protective agent also mixed with the reductant first prior to the reaction will lead to a colloidal gold with dark purple red color and stable under normal conditions for a long time. We measured the particles size in this experiment; it was less than 100 nanometers (Figure 2). It is favorablefor the reductant dispersing in PVP solutions in water and interacting with PVP via the intermolecular force or hydrogen bond. As the amount of PVP increase, which may hamper the reaction between the reductant and $[\mathrm{Au}$ $\mathrm{Cl}_{4}$ ] decreasing, and to producing less Au nuclei in a shorter period, which favors growth of Au nanoparticles, and the larger Au nanoparticles finally [8].

We speculated that the difference of the results might be resulted to the different reducing agents used and PVP and PEG as a protective agent.

\section{REFERENCES}

[1] J. C. Davies, "Managing the Effects of Nanotechnology," Woodrow Wilson International Center for Scholars, Washington DC, 2007.

[2] P. Rodgers, "Nanoelectronics: Single File," Nature Nanotechnology, 2006. doi:10.1038/nnano.2006.5

[3] A. D. McFarland, C. L. Haynes, C. A. Mirkin, R. P. Van Duyne and H. A. Godwin, “Color My Nanoworld,” Journal of Chemical Education, Vol. 81, No. 4, 2004, p. 544A.

[4] C. N. Ramachandra Rao, G. U. Kulkarni P. J. Thomasa and P. P. Edwards, "Metal Nanoparticles and Their Assemblies," Chemical Society Reviews, Vol. 29, No. 1, 2000, pp. 27-35. doi:10.1039/a904518j

[5] V. Sharma, K. Park and M. Srinivasarao, "Colloidal Dispersion of Gold Nanorods: Historical Background, Optical Properties, Seed-Mediated Synthesis, Shape Separation and Self-Assembly," Material Science and Engineering Reports, Vol. 65, No. 1-3, 2009, pp. 1-38. doi:10.1016/j.mser.2009.02.002

[6] D. C. Harris, "Quantitative Chemistry Analysis," 7th Edition, W. H. Freeman, New York, 2006.

[7] Y. W. Tan, X. H. Dai, Y. F. Li and D. B. Zhu, "Preparation of Gold, Platinum, Palladium and Silver Nanoparticles," Journal of Materials Chemistry, Vol. 13, No. 5, 2003, pp. 1069-1075. doi:10.1039/b211386d

[8] H. Liu, Q. Zhou, et al., "Synthesis of Nearly Monodispersive Gold Nanoparticles by a Sodium Diphenylamine Sulfonate Reduction Process,” Journal of Materials Science, Vol. 41, No. 12, 2006, pp. 3657-3662. $\underline{\text { doi:10.1007/s10853-006-6199-2 }}$ 